\title{
EDITORIAL
}

\section{Stoked about Stokes}

\author{
This year marks the 200th anniversary of the birth of George Gabriel Stokes, a physicist and \\ mathematician best known for his contributions to fluid dynamics, but whose work was broader \\ than that.
}

November sees the 72nd annual American Physical Society Division of Fluid Dynamics Meeting. The conference brings together over 3,000 attendees from around the world and covers topics including the fluid dynamics of the interiors of stars, drug transport within the body, and ships and submarines. This variety of topics shows the many research directions and applications of fluid dynamics, a field that is indebted to the work of George Gabriel Stokes, who was born 200 years ago this year.

One of Stokes' most famous scientific contributions is his work on the Navier-Stokes equations, which describe how incompressible fluids flow. In part, the fame of the Navier-Stokes equations derives from the fact that even the basic properties of their solutions are not well understood. A long-standing problem is to prove whether Navier-Stokes equations in 3D always have smooth and globally defined solutions, that is, that they are solved by velocity and pressure functions that can be differentiated again and again without encountering a discontinuity. This problem has stumped mathematicians for years, and is the subject of a US $\$ 1$ million prize from the Clay Mathematics Institute. Although a proof that all solutions are smooth remains elusive, a possible path to a counter-example was highlighted by Terence Tao in a Comment in our July issue, involving 'programming' a fluid to spend a long time near equilibrium but periodically abruptly transition to a rescaled version of itself.

Apart from the interest they garner from mathematicians, the Navier-Stokes equations underlie the whole research area of fluid dynamics. One direction of that field is that of active matter, the study of 'materials' made up of particles that can each convert an energy supply into motion. One way to describe the collective behaviour of these particles is to invoke a new kind of hydrodynamics that includes the 'active stresses' that exist in the system. In a Comment in this issue, Sriram Ramaswamy discusses the history and future of active hydrodynamics, including ways that it may be used to better understand how biological tissues grow and develop.

Another aspect of biology for which fluid dynamics has a role is the study of insect flight. Whether it is a bee, a dragonfly or a mosquito, an insect in flight exerts forces on the fluid of the atmosphere around it and is itself subject to forces from that fluid. However, insects do not simply glide but control their flight. As Itai Cohen and colleagues discuss in a Comment, this control can be both passive - such as in insect wing hinges with mechanical properties that affect how the wing rotates under aerodynamic lift and drag forces - and active, controlled neuronally. Therefore, fully understanding insect flight behaviours requires an understanding of fluids, biomechanics and neuroscience.

On a much larger scale than insect flight, the fluid dynamics of the Earth's atmosphere is experienced by all of us as weather. Weather and climate forecasts are made using numerical simulations of the atmosphere, oceans and icecaps on grids with resolution of tens of kilometres, a scale that is orders of magnitude larger than the length scale set by the viscosity of the fluids. In a Perspective earlier this year, Tim Palmer argued for weather and climate models that treat as stochastic the processes that happen on length scales shorter than the simulation grid. Such models out-perform models that impose a hard cut-off at the length scale of the grid. They also better respect the self-similarity of the NavierStokes equations on all length scales, a symmetry that is incompatible with a hard cut-off length scale.

The breadth of fluid dynamics research is reminiscent of the breadth of the work of Stokes himself. Although he was trained as a mathematician, Stokes made major contributions to physics problems in hydrodynamics and optics, with work spanning theory and experiment. For instance, in the field of optics, he investigated the wave theory of light in the context of diffraction and experimentally identified the phenomenon of fluorescence. Stokes also found ways to apply his research to other fields, contributing to geodesy, for example, by applying his theory for the motion of pendula in fluids to the problem of variations of gravitational acceleration at different points on the Earth's surface.

In many ways, in the years since Stokes lived, scientific research has become more specialized, with researchers focusing on a single topic within a single discipline. Such an approach has certainly paid dividends. However, the interdisciplinary applications of fluid dynamics - and the wide-ranging research career of Stokes himself - show that crossing boundaries can pay off too. 